Archives de sciences sociales des religions

167 | juillet-septembre 2014

Postérités Allemandes

\title{
Compte rendu ASSR
}

\section{OpenEdition}

1 Journals

Édition électronique

URL : http://journals.openedition.org/assr/26198

DOI : 10.4000/assr.26198

ISSN : $1777-5825$

Éditeur

Éditions de l'EHESS

\section{Édition imprimée}

Date de publication : 20 octobre 2014

Pagination : 243-251

ISBN : 978-2-7132-2433-1

ISSN : 0335-5985

Référence électronique

"Compte rendu ASSR », Archives de sciences sociales des religions [En ligne], 167 | juillet-septembre 2014, mis en ligne le 16 décembre 2014, consulté le 24 septembre 2020. URL : http://

journals.openedition.org/assr/26198; DOI : https://doi.org/10.4000/assr.26198

(c) Archives de sciences sociales des religions 
Compte rendu ASSR de Jean Baubérot (1984, no 58/2, p. 277-278).

58.307

LAPLANCHE (François).

L'Evidence du Dieu chrétien. Religion, culture et société dans l'apologétique protestante de la France classique, (1576-1670). Strasbourg, Association des Publications de la Faculté de Théologie Protestante de Strasbourg, 1983, $342 \mathrm{p}$.

La production apologétique protestante française, de 1576 à 1670 , pourrait paraitre modeste : 10 titres contre, par exemple, 94 ouvrages de controverse. Elle a pourtant connu une diffusion supérieure à celle de l'apologétique protestante allemande ou anglaise. Faut-il expliquer ce fait par l'expansion du français, langue de culture européenne? Pas uniquement. Représentant une communauté qui ne groupe que $5 \%$ des habitants du Royaume, les apologètes protestants français ont cependant un succès d'édition plus grand que les apologètes catholiques français. La raison principale de cette réussite est due, selon F.L., au contenu propre de leurs propos qui, seuls, vont “jusqu'au bout de l'élan qui pousse la raison du croyant à justifier son adhésion religieuse» (p. 231). La théologie de la grâce, en renvoyant l'événement de la foi à l'opération du Saint Esprit, n'en est que plus à l'aise pour affirmer le caractère totalement rationnel de l'adhésion de foi. A cela s'ajoute la nécessité, face au catholicisme romain, de défendre la "clarté et suffisance» de l'Écriture.

Selon le discours apologétique réformé il existe une triple évidence de la vérité du christianisme: - Une évidence philosophique et scientifique : épistémologiquement la théologie domine les autres disciplines. La certitude de ses démonstrations reçues directement de Dieu ou déduites de sa Parole contraste avec les contradictions des philosophes, les varia- tions du droit, les conjonctures de la médecine, les incertitudes de la physique; - Une évidence historique: la Bible est antérieure à toute culture et l'hébreu est la mère de toutes les langues. Sans elle l'humanité ne connaîtrait rien de ses origines et ne serait jamais sortie de la barbarie; - Une évidence sociale. La religion conserve et purifie la morale, même si elle n'est pas réductible à cette dernière, or il n'y a pas de lien social durable qui ne suppose un consensus éthique. L'État, par ailleurs, tient son existence et son autorité de Dieu même et qui s'attaque à la religion ruine le fondement de l'État. La situation menacée des réformés français et leur désir de bien se distinguer des protestants régicides anglais les amènent à insister de plus en plus fortement sur ces thèmes.

F.L. estime que l'effort des apologètes réformés comporte un aspect "tragique": le "recul du christianisme dans les élites cultivées s'annonce en négatif dans cette apologétique " (p. 234). On pourrait même globaliser le propos à partir d'une perspective de sociologie de la sécularisation. Le XVIII ${ }^{\mathrm{e}}$ siècle mettra explicitement en cause l'évidence culturelle de la religion chrétienne et le XIX ${ }^{\mathrm{e}}$ son évidence sociale. Bien sûr de telles mises en cause avaient commencé antérieurement -c'est pourquoi une apologétique s'avérait nécessaire - mais comme l'indique l'A. leur pertinence sociale se trouve limitée et on peut encore parler au sujet du XVII ${ }^{\mathrm{e}}$ siècle de "l'évidence du Dieu chrétien ".

Recherche à la fois minutieuse et riche de vastes perspectives, l'étude de F.L. se situe dans la lignée des travaux de L. Febvre et A. Koyré. C'est dire toute son importance.

Jean Baubérot. 
Compte rendu ASSR de Danièle Hervieu-Léger (1994, vol. 88, nº 1, p. 83-84).

88.50

LAPLANCHE (François).

La Bible en France entre mythe et critique (XVI ${ }^{\mathrm{e}}$-XIX ${ }^{\mathrm{e}}$ siècle). Paris, Albin Michel, 1994, 291 p. (Index).

En proposant un ouvrage qui traite de la science des Écritures dans la France moderne, F. L. limite, d'entrée de jeu, l'ambition qu'il poursuit : il ne vise ni à décrire l'ensemble des pratiques de lecture appliquées à la Bible, ni à proposer un manuel exhaustif d'histoire de l'exégèse. Son objectif est de montrer comment l'exégèse biblique s'est progressivement construite, en contexte français, au milieu des conflits culturels qui scandent l'histoire intellectuelle du christianisme occidental, en se confrontant, sur toute la durée de la trajectoire, à deux questions fondamentales, celle, théorique, de la vérité d'une part, celle - directement pratique et politique - de la violence d'autre part. En dix chapitres couvrant trois siècles d'histoire, F. L. dessine le parcours selon lequel le récit biblique passe d'un statut de code global du sens, capable de rendre compte à la fois de la singularité des existences individuelles et de l'ensemble des différences sociales et culturelles, à un état où, assujetti aux règles de la raison, il occupe une place définie dans une histoire des religions.

Ce qui fait tout l'intérêt de cette entreprise, c'est que l'auteur ne se contente pas de retracer les opérations d'une logique scientifique déconstruisant, par étapes, la logique du mythe. Il montre, d'une façon véritablement lumineuse, que ce travail de l'érudition occidentale, qui élabore de nouveaux rapports entre religion et culture, ne peut pas être dissocié des transformations politiques au travers desquelles se redéfinit la fonction religieuse de l'État ou des institutions publiques. Les déplacements de la rationalité accompagnent et rendent possible à la fois la recomposition des dispositifs sociaux d'autorité à partir desquels s'opère la lente déconstruction du mythe chrétien. Ceci ne signifie pas que F. L. rabatte purement et simplement les processus de recomposition du système chrétien sur les déterminations intellectuelles et politiques qui les ont générés. Si la pure question de l'autorité de la vérité entretient, selon ses propres termes, de multiples et souvent troubles rapports avec celle de la vérité de l'autorité, l'émergence de la science de la Bible ne se confond pas, à beaucoup près, avec le réseau de stratégies religieuses ou politiques que son étude permet de mettre à jour. Elle est aussi processus de création culturelle et intellectuelle dans lequel sont réarticulés, de façon effectivement nouvelle, les rapports entre mythe et raison, entre récit et sens, entre histoire et vérité.

L'enquête de F. L. prend pour point de départ la force d'imprégnation extraordinaire du «mythe chrétien», en tant qu'il structure la connaissance et qu'il organise l'espace social dans toute la culture de l'Occident, au titre notamment de l'unité interne que les commentateurs chrétiens prêtent au texte biblique. La Réforme protestante et la Réforme catholique constituent à la fois les entreprises les plus systématiques visant à l'imposition massive de ce mythe à l'Occident et le moment où leur antagonisme même, rencontrant la nécessité pour l'Etat souverain d'assurer son autonomie par rapport à la puissance spirituelle, en induit le premier affaissement. L'immense travail de la critique aux $\mathrm{XVI}^{\mathrm{e}}$ et $\mathrm{XVII}^{\mathrm{e}}$, travail stimulé à la fois par les conquêtes des sciences, par l'enrichissement de la philologie et par les exigences d'une modernité politique en train de se constituer, vise à extraire, dans une problếmatique radicalement nouvelle de la vérité, la portée de ces textes donnés jusque là comme faisant autorité. L'acquis majeur de cette conquête érudite est que la Bible, destinée au peuple hébreu, ne concerne pas directement les chrétiens dans leur existence actuelle : elle les intéresse en tant qu'elle est porteuse d'une théologie et d'une morale dont la vérité s'impose non à travers l'histoire, mais à partir des évidences de la lumière naturelle. Dans cette dissociation entre histoire et vérité, c'est l'ensemble du dispositif chrétien de la certitude qui vacille, et tout l'effort des apologistes protestants aussi bien que catholiques consiste, par des voies différentes, à établir par quels moyens le fidèle peut, dans ce contexte nouveau, accéder à la vérité religieuse. Les turbulences politiques du XVIII ${ }^{\mathrm{e}}$ siècle ouvrent une phase nouvelle de retour au mythe et au symbole, de revalorisation des rapports entre 1'histoire et la vérité, de réhabilitation de la capacité de la langue de cristalliser l'esprit d'un peuple : dans ce mouvement, les religions peuvent être réévaluées, en tant que matrices culturelles des grandes civilisations de l'histoire. Se pose alors aux catholiques comme aux protestants la question de savoir ce qui fonde la place unique qui revient au christianisme en Occident. S'impose ici évidemment l'entreprise du mouvement mennaisien, et son ambition de restituer au mythe chrétien sa capacité de récapitulation universelle de la quête humaine du sens. La confrontation de ces tentatives de restauration au développement d'une science des religions (illustrée en France par Burnouf) méfiante vis-à-vis de toutes les traces d'un impérialisme spirituel possible produit, dans la seconde moitié du XIX ${ }^{\mathrm{e}}$ siècle, le redéploiement d'une apologétique qui place dans la prédication de Jésus et des apôtres, et non plus dans la révélation primitive, le ferment d'une rénovation du mythe chrétien. Des questions nouvelles surgissent : celle des rapports entre l'enseignement de Jésus et le judaïsme de son temps, ou celle des rapports entre l'histoire de l'Église et l'annonce du retour du Christ, questions que l'exégèse chrétienne du 
$\mathrm{XX}^{\mathrm{e}}$ siècle prendra centralement en charge. Sur toute la durée du parcours, le lien est établi entre les remaniements internes de l'exégèse chrétienne, dans ses versions catholiques et protestantes, et les figures transformées de la culture et de la politique dans lesquelles ils s'enracinent. A travers la mise en perspective des processus de circulation des interrogations entre le champ théologique, le champ de la science profane et le champ politique, à travers l'identification des entreprises d'instrumentalisation mutuelle des enjeux présents dans ces différents registres, à travers l'analyse des modalités de la participation des théologiens à l'imaginaire collectif de leur temps, F. L. réalise beaucoup plus que l'exercice de méthode auquel il prétend modestement : celui de faire, pour les historiens, l'histoire d'une discipline théologique. Il ouvre des espaces d'interrogation renouvelés pour tous ceux, historiens, mais aussi sociologues et anthropologues, qui traitent, sur d'autres terrains et avec d'autres outils théoriques, de la construction de l'objet religieux, des rapports entre religion et politique ou des rapports entre religion et culture. La manière discrète et ferme qu'a F. L. de faire avancer son propos ne doit pas tromper: ce livre merveilleusement lisible malgré la technicité du thème, riche d'une érudition impressionnante (dont témoignent abondamment les notes qui accompagnent chaque chapitre), est un livre important, bien au-delà du cercle de spécialistes qu'il concerne en premier lieu.

Danièle Hervieu-Léger. 
Compte rendu ASSR de Bernard Chédozeau (1999, vol. 106, nº 1, p. 123-124).

106.96

LAPLANCHE (François).

Bible, sciences et pouvoirs au XVII ${ }^{e}$ siècle. Naples, Istituto italiano per gli studi filosofici, Bibliopolis, 19, 1997, $145 \mathrm{p}$.

Cet ouvrage réunit les textes de conférences sur \& les rapports entre l'heritage biblique et la philosophie occidentale $*$.

Dans "L'histoire de l'exégèse biblique au XVII ${ }^{\mathrm{c}}$ siècle», F.L. étudie la naissance de la philologie, puis les modes de son affirmation dans l'exégèse biblique (Érasme, les hébraistes chrétiens) à côté de l'autoritế de la Tradition $\left(X V I^{e}\right.$ siècle). En une sorte de va-et-vient entre les textes et leur histoire, la Bible est peu à peu étudiée à la façon des textes profanes, et il en surgit deux problématiques fondamentales: le statut même de la Bible, chez les catholiques et chez les protestants, et le problème des sens littéral (ou grammatical, qui est désormais au cour des controverses), et spirituel (allégorique, tropologique, anagogique ou mystique) de l'Écriture. Les rapports entre religion et politique (sont-elles autonomes l'une par rapport à l'autre?) en sont renouvelés; dans le domaine de la cosmologie, les théologiens cherchent encore à faire d'abord confiance à la Bible. Au fül du siècle suivant, le recul des interprétations symboliques et mystiques conduit les exégètes critiques comme R. Simon à privilégier largement le sens litcéral $($ Le sens littéral et ses problèmes: l'éloignement de l'origine $»)$ : l'auteur en étudie les conséquences : «La Bible est entrée dans le temps» (p. 67). F.L analyse ensuite les relectures politiques de la Bible qu'imposent les événements tragiques du XVII ${ }^{\mathrm{e}}$ siècle (droit à la résistance, devoir de soumission au pouvoir établi, source du pouvoir, distinction des pouvoirs politique et religicux, par exemples. Dans « La Bible et la vérité $\%$, il considère quelques-uns des problèmes que les nouvelles science et philosophic soulèvent dans l'interprétation biblique, les formes que revêtent les premiers conflits; l'avènement de la science soulève la difficile question «sens vrái du texte et verrité de la chose" (p. 98). En guise de bilan ( " De l’histơire à l'interprétation $\%$ ): si on reconnât le jeu de l'histoire dans la Bible, comment concilier Révélation et raison, et que devient le livre sacré? On assiste alors à une récévaluation des sens symboliques et mystiques el de l'exégèse spirituel]e. Finalement F.L. conclut, de façon d'ailleurs nuancée, à la aperte de crédibilité de la Bible " au XVII ${ }^{e}$ siècle.

On retrouve les qualités d'un auteur savant et d'un pédagogue d'une limpide clarté dans ce livre qui témoigne du rôle capital qu'ont joué les lectures et interprétations de la Bible aux $\mathrm{XVI}^{\mathrm{e}}$ et $\mathrm{XVII}^{\mathrm{e}}$ siècles.

Bernard Chédozeau. 
Compte rendu ASSR de Pierre Lassave (2006, vol. 134, p. 221-225).

\title{
La crise de l'origine, la science catholique des Évangiles et l'histoire au xxe siècle
}

\author{
Paris, Albin Michel, coll. "L'évolution de \\ I'humanité », 2006, 707 p.
}

Cet épais volume fait suite à un précédent de l'auteur sur la naissance et les tribulations de la critique historique des écritures saintes, La Bible en France entre mythe et critique, $\mathrm{XVI}^{e}-\mathrm{XIX}^{e}$ siècles (Paris, Albin Michel, 1994), livre paru dans la même prestigieuse collection d'histoire fondée au début du siècle par Henri Berr. Mis bout à bout, ces deux ouvrages brossent un tableau détaillé et de longue durée des thèmes, des acteurs et des conflits liés à la lente conversion du fondement scripturaire de la principale religion du pays en objet de connaissance scientifique. La « crise moderniste », point d'aboutissement du premier volume, est le point de départ du second (dans la même collection, le titre désormais classique d'Émile Poulat, Histoire, dogme et critique dans la crise moderniste - Paris, Albin Michel, 1962 -, pourrait faire charnière entre les deux).

François Laplanche s'explique d'abord sur son titre : la « science catholique », désigne depuis Lamennais le corps de connaissances que développe l'Église romaine à partir de la Bible sur l'origine et les fins de la vie humaine (création du monde, péché originel, promesse de salut, nécessité du sacrifice, etc.). Savoir traditionnel et dogmatique que la Réforme puis la raison des Lumières ont remis en question. Après Galilée, Newton et Darwin, la science catholique a dû en effet faire front aux progrès de l'histoire laïque des religions qui relit les écritures saintes à l'aune de leur complexe élaboration dans les flux mêlés des civilisations antiques. $\mathrm{La}$ « crise de l'origine », titre principal de l'ouvrage, souligne la transformation par la raison critique du dépôt originel en une bibliothèque de récits mythiques, mystiques ou moraux aux significations fluctuantes au gré des conjectures textuelles et des découvertes archéologiques. Mais la disparition de la science catholique qui se joue après la crise moderniste ne signifie pas pour autant que l'Église romaine a perdu définitivement la partie de l'exégèse biblique face à la « science indépendante » et ses alliés libéraux, protestants et juifs. Malgré le poids des siècles de croyance en son infaillibilité, l'institution s'est en effet adaptée au nouveau cours des sciences historiques en reconnaissant progressivement les travaux de ses propres érudits progressistes qu'elle avait d'abord commencé par mettre à l'index.

De la loi de séparation des Églises et de l'État jusqu'aux années 1980 en France, l'historien retrace par le menu les résistances romaines face aux conquêtes de la «science des religions »; il développe la lente percée des cleres les plus savants qui réussissent non sans revers douloureux à infléchir le magistère en faveur d'une lecture historique répondant aux exigences modernes de l'intelligence de la foi ; il aborde, enfin, un ensemble de nouvelles questions posées par le devenir incertain d'une science des écritures saintes qui a pourtant gagné en autonomie. Au fil de l'enquête, la question des origines du christianisme devient centrale et fournit matière aux rapprochements entre interprétations catholiques, protestantes, juives et agnostiques. Reprenons succinctement ce fil.

Au tournant du siècle dernier, la guerre des deux France traverse l'exégèse. Depuis Burnouf et Renan, les progrès de l'histoire comparée des religions soutenue par l'élite républicaine (création de la section des sciences religieuses à l'École pratique des hautes études, chaires d'histoire des religions en Sorbonne et au Collège de France) révoquent en doute la science catholique. Le Vatican se cabre malgré les ouvertures de sa dernière 
encyclique (Providentissimus, 1893). Sous l'effet de pressions réactionnaires, sa Commission biblique prend une allure inquisitoriale. Pour avoir rigoureusement déconstruit les écritures néotestamentaires en révélant la constitution ecclésiologique de leur message, l'abbé Alfred Loisy (1857-1940) est excommunié en 1908. Pourtant farouchement opposé au radicalisme critique de Loisy, le dominicain Marie-Joseph Lagrange (1855-1938), fondateur de l'École de Jérusalem, voit ses travaux pionniers mis à l'index. Autour de ce dernier, les réseaux de résistance de l'exégèse catholique s'organisent entre Jérusalem, Toulouse, Paris et Lyon pour faire patiemment avancer leur programme de recherche des liens entre l'inspiration du texte et sa forme plurielle et littéraire, programme mobilisant des méthodes analytiques qui préparent à accueillir plus sereinement les avancées de l'école allemande (Formgeschichte, histoire des formes) et bientôt de l'anthropologie des religions sur la scène universitaire.

Après le drame de la Grande Guerre, les positions s'affirment en face à face entre exégètes catholiques et historiens universitaires au fil d'œuvres marquantes que l'auteur revisite de près. Entré au Collège de France après son excommunication, Loisy s'attaque ainsi à la question débattue des liens entre mythe et rite en démontrant que la reconstruction christologique forgée par le Nouveau Testament dans la diversité de ses voix et de ses emprunts aux religions à mystères transcende une communauté de foi primitive que l'analyse textuelle décèle à travers les paroles déconcertantes de Jésus. Le partage fraternel du pain par Jésus selon un rite de table antique devient culte et dogme (l'eucharistie) après la résurrection du Christ. Dans le même cercle de la Société Ernest Renan, Charles Guignebert (1867-1939), libre penseur et professeur en Sorbonne, ajuste la focale au contexte socioculturel de ces transferts symboliques. Maurice Goguel (1870-1955), professeur à la faculté protestante de Paris montre de son côté comment l'édifice doctrinal et imaginaire du christianisme s'ancre dans un donné historique que les contradictions intertextuelles authentifient paradoxalement. Aiguillonnés par cette « exégèse indépendante » en plein essor, les érudits catholiques livrent un combat apologétique vigoureux qui va faire sortir l'Église de l'impasse intellectuelle où l'intransigeance pontificale l'avait conduite. À rebours de la mise en doute générale de l'historicité des évangiles, Lagrange établit l'ancienneté de leur tradition christologique et refuse d'y voir le produit tardif de la seconde génération chrétienne et, encore moins, un sousproduit des religions à mystères. Les jésuites les plus libéraux emboîtent le pas du savant dominicain : dans des publications de synthèse « alertes et claires », Léonce de Grandmaison (1868-1927), avance ainsi l'équation de la proportion entre la cause (la réalité de Jésus-Christ) et l'effet (l'expansion du christianisme).

L'entre-deux-guerres est, dès lors, sous-tendu par le combat entre universitaires qui excipent de l'analyse intertextuelle la dimension mythologique des origines du christianisme et clercs qui, en utilisant des méthodes voisines, concluent à l'inverse à l'historicité extraordinaire de Jésus, de son message et de la foi qu'il engendre. Les années 1930, de crise économique et morale, sont aussi marquées par la révolution théologique du luthérien Rudolf Bultmann (1884-1976). Ce dernier admet d'autant plus les évangiles comme système de reconstructions mythologiques que leur message central, le kérygme, leur échappe paradoxalement en s'adressant à l'homme intemporel. Reconnaître l'interpellation «existentiale » de Jésus neutralise et même rend stérile le vieil antagonisme entre mythe et histoire. La légende, aussi irréelle soit-elle, peut être plus vraie que l'histoire. C'est ce que font par ailleurs valoir sur d'autres aires culturelles les travaux de la nouvelle histoire des représentations collectives, l'école des Annales influencée par la sociologie durkheimienne et l'anthropologie anglo-saxonne.

À la veille de la Seconde Guerre et de la tragédie de la Shoah, la quête des origines du christianisme investit les liens complexes qui le rattachent au judaïsme. L'exégèse érudite et 
réflexive initiée par Lagrange trouve ses relais jusque dans l'enseignement de l'Institut biblique pontifical. En 1943, l'encyclique Divino Afflante Spiritu lui fait enfin droit en reconnaissant l'infirmité humaine des écritures saintes justifiée pour la foi par la condescendance divine et pour la raison par leur fonction apologétique. S'ouvre après guerre une nouvelle ère d'études, de fouilles, de traductions et de rapprochements entre perspectives confessionnelles et scientifiques. Les générations d'exégètes nés dans le premier quart du siècle et formés à la «méthode historico-critique » vont donner toute leur mesure avec la libéralisation confirmée par le concile Vatican II. La découverte des manuscrits de la Mer Morte à Qumran, en 1947, relance l'étude interdisciplinaire et internationale sur la pluralité des communautés du judaïsme pré-chrétien et du judéo-christianisme. Confrontée aux aspirations nationales des peuples colonisés et aux espérances temporelles nourries de visions marxistes, l'Église catholique ne peut rester arc-boutée sur sa condamnation du monde moderne. Comme l'écrit l'auteur : "Désireux de s'éclairer davantage sur la plus ancienne théologie missionnaire, celle du Nouveau Testament, les catholiques deviennent plus aptes à recevoir la conclusion fondamentale de l'École des formes, conclusion selon laquelle les Évangiles ne retracent pas la biographie de Jésus, mais développent chacun à sa manière le message pascal, dans une perspective catéchétique adaptée à chaque auditoire : le Jésus de l'histoire est inséparable du Christ atteint par la "foi de Pâques". De plus, la convergence des recherches d'ecclésiologie autour d'une conception moins juridique de l'Église, plus communautaire et plus mystique, rend acceptable le rôle de la communauté dans la formulation de la foi primitive. » (p. 418).

Le combat exégétique n'a pas qu'une portée étroitement théologique mais concerne pratiquement l'Église dans son organisation même, sa liturgie, sa mission pastorale et plus généralement sa présence au monde. Les difficultés rédactionnelles de la constitution conciliaire Dei Verbum (1965) rappellent cependant que la voie moyenne entre l'antihistoricisme de Bultmann et l'histoire sainte des intégristes n'a rien d'un « long fleuve tranquille » comme l'écrit l'auteur. La question de la « seconde quête du Jésus de l'histoire », ce corps de significations que l'on déduit de l'histoire des traditions inscrites dans les strates rédactionnelles, fait débat entre pairs. Contre le dualisme bultmanien du mythe et du sens, Oscar Cullmann (1902-1999), observateur protestant du concile, fait valoir l'épaisseur temporelle de l'histoire du salut. La recherche sur la rédaction du Nouveau Testament, ses soubassements communautaires multiples et concurrents ne s'épuise pas comme en témoignent les travaux remarqués du jésuite Xavier Léon-Dufour (né en 1912). L'exégèse fait œuvre œcuménique et s'affirme comme groupe scientifique au-delà des frontières confessionnelles. Le père Pierre Grelot (né en 1917), « combattant de la Bible en Église », contribue ainsi à la création en 1966 de l'Association catholique française pour l'étude de la Bible (ACFEB), suivie en 1967 de la Bibliothèque œcuménique et scientifique d'études bibliques (BOSEB). Nous n'irons pas jusqu'à dire comme l'auteur qu'une nouvelle profession est née, mais l'ACFEB réunit un milieu hybride - entre science, théologie et pastorale - qui affirme son autonomie. Caisse de résonance de multiples événements comme l'introduction des méthodes sémiotiques, la traduction œcuménique (TOB) ou l'expertise ecclésiologique (par exemple, la question du célibat et de la masculinité des prêtres), l'association voit s'estomper les conflits doctrinaux entre exégètes et hiérarchie romaine. Ses liens avec la recherche universitaire s'intensifient ; elle coopte dans ses rangs des spécialistes reconnus hors de toute obédience confessionnelle, contribuant de la sorte à un début de féminisation du milieu.

Une vingtaine d'années après Vatican II, « une page se tourne » comme l'écrit l'auteur. Un socle cognitif commun transcende les divisions anciennes ; par exemple, il est désormais exclu que les évangiles soient l'œuvre de témoins directs et une batterie de critères de reconnaissance de la fiabilité des sources fait l'unanimité (multiples attestations, cohérence, 
dissemblance, explication suffisante). Le rapprochement entre exégèse et sciences humaines apporte à ses dernières de nouveaux terrains d'étude, comme c'est le cas pour la sémiotique ou la psychanalyse, et en retour interpelle le bibliste sur le lieu épistémologique de sa lecture comme l'a montré Michel de Certeau (1926-1986) mettant en cause la «bulle » positiviste, élitiste et passéiste d'une certaine érudition. La sécularisation contemporaine relance ainsi pour l'exégèse la question de la réception et partant fait reconnaître les écritures saintes comme actes de langage qui engagent une chaîne d'agents aux intentions et horizons distincts que toute lecture met en présence dans une situation culturelle donnée. L'œuvre du jésuite Paul Beauchamp (1924-2001) ressaisit ainsi la diversité du corpus biblique à travers un prisme téléologique commun (de la loi au don et au pardon, de l'élection d'un seul, le peuple juif, à l'accueil de tous depuis le Christ, de la mort à la vie) qui renvoie à sa fonction d'interpellation pour chacun. Plus généralement, l'histoire des effets du texte (Wirkungsgeschichte) cultivée en Allemagne fait ainsi système avec l'analyse de ses composants littéraires développée outre atlantique dans les Cultural studies.

Au terme de cette histoire intellectuelle mouvementée, l'auteur tente un premier bilan. En un siècle, la science catholique des écritures saintes a disparu en tant que telle. La raison a séparé les régimes de vérité entre ce qui relève du sens de l'existence humaine, des traditions ecclésiales ou des représentations imaginaires de l'origine du monde. À la double encontre du littéralisme conservateur et de l'historicisme moderniste, une première génération de savants a su faire valoir dans l'entre-deux-guerres la complexité des sources et ouvrir un espace interprétatif pluriel qui ne pouvait réduire les livres canoniques à leur seule antiquité. Prise dans la crise de conscience occidentale sur fond de révolution scientifico-technique et de périls totalitaires, l'Église romaine ne pouvait sauver une part de son magistère qu'en faisant droit à cette exégèse progressiste talonnée sur sa gauche par une histoire profane des religions en plein essor. Une véritable révolution théologique en découle que la génération suivante d'exégètes va s'efforcer d'instruire après la Seconde Guerre : l'axiome déductif selon lequel la Bible est parfaite parce que Dieu est son auteur se renverse en effet en question inductive : comment cette collection de livres, évidemment écrits par des hommes, peut-elle être le lieu possible d'une révélation transcendante ? « La question de l'origine se voit ainsi formulée autrement : il ne s'agit plus de reconstituer une chaîne de témoins historiques à la manière de l'apologétique ancienne, mais de décrire, en ses divers chemins, la capacité créatrice du témoignage apostolique » (p. 594). Chemins multiples de l'analyse qui conduisent les savants catholiques à nouer des liens horizontaux avec les spécialistes d'autres traditions exégétiques (protestante et juive), à coopérer avec des historiens universitaires saisis par le linguistic turn (tout événement s'éprouve dans les jeux de langage), et à se laisser interpeller en retour par la philosophie herméneutique (Gadamer, Lévinas, Ricœur).

La pluralité conviviale de l'exégèse qui apparait en fin de parcours débouche sur un paysage plus professionnel que confessionnel, mais aux réseaux disciplinaires et affinitaires éclatés sur une scène toujours plus internationale. Les relations ou les tensions entre les institutions universitaires, confessionnelles, professionnelles et éditoriales mériteraient d'ailleurs à elles seules une étude sociologique qui dépasse l'horizon historiographique de l'ouvrage, largement surdéterminé comme on l'a vu par l'autonomisation de l'exégèse au sein de l'Église romaine. Se faisant l'écho d'un sentiment général de délocalisation - « Nous sommes ailleurs » - qui s'est emparé des praticiens postconciliaires, l'auteur ne pointe pas moins un intéressant double mouvement contradictoire dans lequel ceux-ci et leurs successeurs se trouvent aujourd'hui pris : d'un côté, un recentrage ecclésial (édifier les divers lecteurs dans la foi comme le demande l'institution), et de l'autre, un décentrement public obligé par les multiples investissements scientifiques, communautaires, esthétiques, médiatiques, dont la Bible fait aujourd'hui l'objet 
dans un monde globalement sécularisé mais troublé par les retours identitaires aux traditions religieuses. Les dilemmes de conscience et les bifurcations d'itinéraires que cette hétérotopie suscite (nombre d'exégètes se sont rendus à l'état laïque pour sauvegarder leur liberté de recherche) ouvrent ici également sur une plus vaste enquête.

Revenant sur son volume précédent, l'auteur considère que l'exégèse et l'histoire des dogmes ont détrôné le thomisme du XIX ${ }^{\mathrm{e}}$ siècle, doctrine centrale de synthèse entre le traditionalisme et le cartésianisme, qui plaçait l'Église en porte-à-faux avec son temps. L'étude critique des sources n'a pas seulement ravalé l'Église à son rang d'institution historique, facilitant par là même son ouverture au monde moderne, mais elle a aussi intériorisé le sens de sa vocation à travers une interprétation des écritures saintes qui assume sa propre finitude et respecte les autres interprétations présentes et passées. Il cite à cet égard, et pour finir, Paul Ricœur (1913-2005), en hommage à sa mémoire : « Si vraiment les religions doivent survivre, elles devront satisfaire à de nombreuses exigences. Il leur faudra en premier lieu renoncer à toute espèce de pouvoir autre que celui d'une parole désarmée. Elles devront en outre faire prévaloir la compassion sur la raideur doctrinale ; il faudra surtout - et c'est le plus difficile - chercher au fond même de leurs enseignements ce surplus non-dit grâce à quoi chacune peut espérer rejoindre les autres, car ce n'est pas à l'occasion de superficielles manifestations, qui restent des compétitions, que les vrais rapprochements se font : c'est en profondeur seulement que les distances se raccourcissent. » (p. 604). Par cette ultime citation à tonalité normative F. Laplanche indique que l'histoire intellectuelle qu'il vient de nous retracer peut avoir un sens souhaitable, par exemple ce « possible retour aux Écritures sans fondamentalisme » qui semble avoir ses faveurs.

Le récit découpé en douze chapitres est accompagné en fin de volume d'utiles notices biographiques sur les principaux acteurs de cette histoire côté catholique et francophone. Nous n'en avons évoqué ici que quelques-uns, mais cette histoire très documentée décrit une scène fort dense où les acteurs d'arrière-plan, émissaires de palais pontificaux comme le philosophe Jean Guitton, jouent un rôle non négligeable dans les changements ou les blocages survenus. Ces notices finales auraient pu intégrer les influents exégètes ou théologiens protestants et de langue allemande ou anglaise rencontrés au fil du livre sans parler des historiens et spécialistes de la Bible inscrits dans la laïcité scientifique ou universitaire. Il y faudrait sans doute un dictionnaire. La focale mise sur l'exégèse catholique de langue française, qui justifie amplement le choix éditorial, n'en débouche pas moins sur un espace intellectuel et institutionnel qui s'est diversifié au cours des deux dernières décennies et qui pose aujourd'hui une série de questions. Il en est ainsi de la pluralisation des paradigmes d'interprétation qui sape l'autonomie relative de l'exégèse. Il en est aussi du renouvellement des générations qui s'avère problématique dans une Église en proie à la perte des vocations; renouvellement ou déclin de l'exégèse qui ne peut s'appréhender indépendamment des formations universitaires et des autres traditions de lecture moins atteintes par le temps.

Riche d'informations de première main, ce second volume, écrit dans une langue limpide, s'avère précieux. Par la connaissance à la fois globale et fine qu'il délivre de tout un pan de l'histoire intellectuelle du catholicisme français. Par les questions vives qu'il soulève sur les liens entre sciences humaines et écritures saintes à la frontière des institutions religieuses et universitaires dans un contexte de modernité avancée.

Pierre Lassave. 
